\title{
Spontaneous quorum-sensing hierarchy reprogramming in Pseudomonas aeruginosa laboratory strain PAO1
}

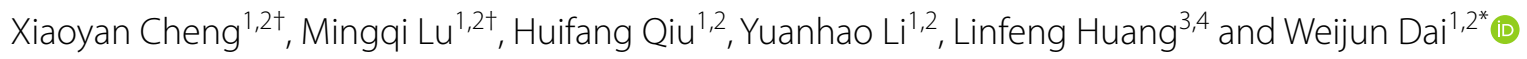

\begin{abstract}
Pseudomonas aeruginosa strain PAO1 has been commonly used in the laboratory, with frequent genome variations reported. Quorum sensing (QS), a cell-cell communication system, plays important role in controlling a variety of virulence factors. However, the evolution and adaptability of QS in those laboratory strains are still poorly understood. Here we used the QS reporter and whole-genome sequencing (WGS) to systematically investigate the QS phenotypes and corresponding genetic basis in collected laboratory PAO1 strains. We found that the PAO1-z strain has an inactive LasR protein, while bearing an active RhI QS system and exhibiting QS-controlled protease-positive activity. Our study revealed that an 18-bp insertion in mexT gene gave rise to the active QS system in the PAO1-z strain. This MexT inactivation restored the QS activity caused by the inactive LasR, showing elevated production of pyocyanin, cyanide and elastase. Our results implied the evolutionary trajectory for the PAO1-z strain, with the evulutionary order from the first Las QS inactivation to the final Rhl QS activation. Our findings point out that QS homeostasis occurs in the laboratory P. aeruginosa strain, offering a potential platform for the QS study in clinical isolates.
\end{abstract}

Keywords: Quorum sensing, LasR, MexT, Pseudomonas aeruginosa, Bacterial communication

\section{Introduction}

Pseudomonas aeruginosa is an opportunistic pathogen that causes several severe acute and chronic human infections, including the infections in cystic fibrosis (CF) patients with compromised immune systems (Gellatly and Hancock 2013; Klockgether and Tümmler 2017). A variety of $P$. aeruginosa virulence factors are regulated by the quorum-sensing (QS) system (Papenfort and Bassler 2016). QS is a bacterial cell-cell communication system that controls the expressions of hundreds of genes in $P$. aeruginosa. Two acyl-homoserine lactone (AHL) QS systems, LasI-LasR and RhlI-RhlR, were identified in P. aeruginosa. LasI and RhlI catalyze the productions of

\footnotetext{
*Correspondence: daiweijun@scau.edu.cn

${ }^{\dagger}$ Xiaoyan Cheng and Mingqi Lu contributed equally to this work

${ }^{2}$ Guangdong Province Key Laboratory of Microbial Signals and Disease

Control, Integrative Microbiology Research Center, South China

Agricultural University, Guangzhou 510642, China

Full list of author information is available at the end of the article
}

diffusible QS signal N-3-oxododecanoyl homoserine lactone (3OC12-HSL) and butyryl-HSL (C4-HSL), respectively. 3OC12-HSL-bound LasR activates the expression of downstream genes. C4-HSL-bound RhIR acts analogously to 3OC12-HSL-bound LasR. The activation of Rhl QS system requires the induction of the Las QS system. These two AHL QS systems also interact with a Pseudomonas Quinolone Signal (PQS) system. In general, the LasI-LasR is atop the QS hierarchy and deletion of LasI or LasR results in the inactivation of the whole QS system (Lee and Zhang 2015).

MexT is a transcriptional regulator of the LysR family and positively controls the MexEF-OprN efflux pump (Köhler et al. 1999; Maddocks and Oyston 2008). This efflux pump is related to the increased resistance of chloramphenicol, trimethoprim and fluoroquinolones (Köhler et al. 1997a, b). As a global transcriptional regulator, in addition to regulation of the mexEF-oprN operon and a neighboring gene mexS (Köhler et al. 1999), MexT 
also regulates the expressions of more than 40 genes (Tian et al. 2009a). Because MexEF-OprN pump exports the PQS precursor HHQ (Lamarche and Déziel 2011), MexT regulates many QS-controlled phenotypes. Inactivation of MexEF in the background of the LasR mutant also elevates the QS-controlled pyocyanide production (Kostylev et al. 2019). On the other hand, overexpression of MexT leads to the attenuated QS-controlled phenotypes, such as the production of pyocynin, C4-HSL, cyanide, elastase and rhamnolipids (Köhler et al. 2001; Tian et al. 2009b).

Mutations in the mexT gene have been reported in clinical isolates from CF patients (Smith et al. 2006), intestinal tissues (Gilbert et al. 2012), and commonly used laboratory PAO1 strains (Luong et al. 2014; Maseda et al. 2000). Inactivation of MexT in those strains exhibited increased pyocyanin production, high swarming motility, reduced chloramphenicol resistance and increased destructive capability on tissues (Gilbert et al. 2012; Luong et al. 2014). Mutations in mexT gene were also found in the PAO1 LasR mutant grown in protein-based broth (Oshri et al. 2018), and supplementation of synthetic C4-HSL or co-culture with clinical isolates secreting C4-HSL can greatly accelerate this genetic adaptation (Kostylev et al. 2019).

The $P$. aeruginosa strain PAO1, obtained from a wound in Melbourne, Australia (Holloway and Morgan 1986; Holloway 1955), serves as a reference strain commonly used for Pseudomonas research in laboratories worldwide. Laboratory-propagated and -maintained PAO1 strains have been found to contain genome variations (Chandler et al. 2019; Klockgether et al. 2010), while QS characteristics in different laboratory PAO1 strains remain illustrative. To address this concern, we systematically surveyed QS profiles in three PAO1 strains derived from different sources. We used skim milk plate and QS reporter assays to screen QS phenotypes in laboratory PAO1 strains. Our study found that the PAO1-z strain showed reduced QS activity when compared to the other two laboratory PAO1 strains. Further genetic analysis revealed that the lasR gene in the PAO1-z strain was disrupted by a 3-bp insertion, resulting in a nonfunctional LasR protein and an impaired Las QS system. The WGS analysis further revealed the mexT gene, which encodes a global transcriptional regulator, has an 18-bp deletion mutation. Although the PAO1-z strain contains a nonfunctional LasR, the Rhl QS system in PAO1-z is activated by the MexT inactivation, producing elevated Rhl-regulated products such as pyocyanin, cyanide and elastase. Our findings revealed that QS adaptation occured in the laboratory PAO1 strain, which probably underwent a QS evolution scenario that an impaired Las
QS system was followed by the activation of the Rhl QS system.

\section{Materials and methods \\ Bacterial strains and growth}

Pseudomonas aeruginosa PAO1-u strain was obtained from E. Peter Greenberg (University of Washington, USA), PAO1-m strain from Matthew Parsek (University of Washington, USA), and PAO1-z strain was collected from Zhang Lianhui (SCAU, China). The PAO1 strains and the mutant derivatives were grown in Luria Bertani (LB) broth containing $10 \mathrm{mg} / \mathrm{ml}$ tryptone, $5 \mathrm{mg} / \mathrm{ml}$ yeast extract, $10 \mathrm{mg} / \mathrm{ml} \mathrm{NaCl}$ at $37{ }^{\circ} \mathrm{C}$. Unless otherwise specified, $P$. aeruginosa strains were cultured in 14-mm FALCON tubes (Corning, USA) containing $3 \mathrm{~mL}$ medium, with shaking (225 RPM) at $37^{\circ} \mathrm{C}$. Escherichia coli was grown in LB broth at $37^{\circ} \mathrm{C}$. The bacterial strains used in this study are listed in Additional file 1: Table S3.

\section{Construction of $P$. aeruginosa mutants}

Either point mutation or full gene knocking out was based on the homologous recombination exchange approach as described previously (Rietsch et al. 2005). Briefly, about 500 1000 bp of DNA flanking the targeted single nucleotide substitution or full length of gene of interest were PCR-amplified and cloned into pEXG-2 vector (Gentamycin resistance, $\mathrm{Gm}$ ) (Rietsch et al. 2005; Stover et al. 2000) with the Vazyme ClonExpress II One Step Cloning kit (Vazyme Biotech, Nanjing, China), generating pEXG-flanking constructs. The primers used for cloning are listed in Additional file 1: Table S2. The pEXG-flanking construct was mobilized into $P$. aeruginosa strain by triparental mating with the help of $E$. coli PRK2013 strain (Kanamycin resistance, Km). Point mutation or full-length gene deletion mutants were first selected on Pseudomonas Isolation agar (PIA) containing Gm100 and further selected on LB agar containing 10\% sucrose. All mutants were confirmed by PCR amplification and subsequent DNA Sanger sequencing.

\section{Constitutive expression of an extra copy of mexT}

Open reading frame sequences of gene mexT were fused with 265 bp native promoter region sequence and cloned into pUC18T-mini-Tn7T-Gm (NCBI accession number: AY599232) (Choi and Schweizer 2006a), generating miniTn7-mexT. The miniTn7-mexT was integrated into the neutral site of genome of PAO1 strains together with the transformation of helper plasmid pTNS2 (NCBI accession number: AY884833). Integration event was confirmed by PCR amplification and DNA sequencing. The excision of $\mathrm{Gm}$ resistance was performed with pFLP2 
plasmid (NCBI accession number: AF048702) (Choi and Schweizer 2006b) and selected on LB agar containing 5\% sucrose.

\section{QS reporter assay}

PlasI-GFP and PrhlA-GFP (Feltner et al. 2016) were used to quantify the LasR- and RhlR-responsive activities, respectively. PlasI-GFP and PrhlA-GFP were mobilized into PAO1 strains and selected on LB agar plate $(\mathrm{Gm})$. PAO1 strains bearing QS reporter plasmids were grown in LB broth containing $50 \mathrm{mg} / \mathrm{mL}$ gentamycin for $12 \mathrm{~h}$. Cell pellets were washed with PBS and subjected to microplate reader (Synergy H1MF, BioTek Instruments, USA) for GFP measurement.

\section{Skim milk assay}

Total proteolytic activity of $P$. aeruginosa strains was evaluated through the skim milk assay, in which the tested strains form a zone of clearing on skim milk agar plate. Individual colonies were spotted on the skim milk agar plates $(25 \% \mathrm{LB}, 4 \%$ skim milk, $1.5 \%$ agar). The protease-catalyzed zones were photographed after incubation at $37^{\circ} \mathrm{C}$ for $18 \mathrm{~h}$.

\section{Pyocyanin production}

Overnight cultures of $P$. aeruginosa grown in LB broth were diluted into $4 \mathrm{~mL} \mathrm{LP}$ medium $(20 \mathrm{~g} / \mathrm{L}$ Gelation peptone, $1.4 \mathrm{~g} / \mathrm{L} \mathrm{MgCl}_{2}, 10 \mathrm{~g} / \mathrm{L} \mathrm{K}_{2} \mathrm{SO}_{4}, 10 \mathrm{mM}$ Glycerol, $\mathrm{pH} 7.2$ ) to reach a starting $\mathrm{OD}_{600} \approx 0.02$ and spotted on to the LP agar plate for visualization.

\section{Cyanide measurement}

Cells grown in LB broth overnight were diluted to OD $600 \approx 0.1$ and spotted on to the peptone agar plate ( $2 \%$ peptone in $1.5 \%$ agar). The plates were covered with filter paper soaking with $5 \mathrm{mg} / \mathrm{mL}$ Copper (II) ethylacetoacetate and $5 \mathrm{mg} / \mathrm{mL}$ 4,4'-methylenebis- (N,Ndimethylaniline) and incubated at $37^{\circ} \mathrm{C}$ for $18 \mathrm{~h}$.

\section{Elastase production assay}

$P$. aeruginosa strains grown in $\mathrm{LB}$ broth overnight were diluted to OD600 $\approx 0.02$ in $2 \mathrm{~mL}$ LB broth for shaking at $37^{\circ} \mathrm{C}$ for $8 \mathrm{~h}$. The cells were spin down at $16,000 \mathrm{~g} \times 2 \mathrm{~min}$ and $500 \mu \mathrm{l}$ supernatants were transferred to a tube containing the same amount of ECR buffer $(0.1 \mathrm{M}$ Tris-Cl, $1 \mathrm{mM}$ Cacl $2,5 \mathrm{mg} / \mathrm{mL}$ Congo red, $\mathrm{pH} 7.2$ ) at $37^{\circ} \mathrm{C}$ for $2 \mathrm{~h}$. Reaction was stopped by adding $100 \mu \mathrm{l}$ EDTA $(0.12 \mathrm{nM})$. Cells were pelleted at $5000 g \times 5 \mathrm{~min}$ and supernatants were measured at OD495 nm.

\section{Quantitative real-time PCR (qRT-PCR)}

Total RNA of PAO1 strains and derivatives were reverse transcribed using the HiScript II 1st Strand cDNA Synthesis Kit (Vazyme Biotech, Nanjing, China) following the manufacturer's instructions. The obtained cDNA was used for qRT-PCR. Quantification reactions containing SYBR qPCR Master Mix (Vazyme Biotech, Nanjing China) were prepared in 96-well plates and run in StepOnePlus Real-Time PCR System (Applied Biosystems, USA) as recommended. Primers used for qRTPCR are listed in Table S2. The expression of targets of interest was normalized to the expression level of the proC gene. Reactions were performed in triplicate.

\section{Whole-genome sequencing by Illumina HiSeq}

$1 \mu \mathrm{g}$ of microbial genomic DNA was sonicated to an average size of $\sim 350$ bp by Covaris-S220 ultrasonicator (Covaris, Woburn, MA, USA). Illumina DNA fragment library preparation was performed following the manual of Next-Generation Sequencing DNA library preparation kit (Novagen). Briefly, the fragmentated DNA products were end repair and ligated with an adapter. Adapter-ligated products were purified using AMPure XP beads (Agencourt-Berkman Coulter, USA) and enriched through PCR amplification using the custom adapter-specific primers. The obtained unbiased short read library was further cleaned up with AMPure XP beads. Pair end Illumina HiSeq PE150 sequencing was performed with an Illumina Novaseq 6000 sequencing system.

\section{Analysis of Illumina HiSeq short reads}

Raw short reads were subjected to quality control including removing adapters using cutadapt (v1.16) by Novagen (Novagen, China), yielding clean short reads. Clean short reads were mapped to the PAO1 reference genome (accession number NC_002516.2) with bwa (v0.7.15r1140). The mapped short reads were subjected to a genome-wide genetic variant calling using Samtools (v1.5) and Breakdancer (v1.4.5) software. The statistics of short read analysis is listed in Additional file 1: Table S1.

\section{Used software}

Following bioinformatics software were used in this study:

Cutadapt, version 1.16 (https://github.com/marcelm/ cutadapt/),

Bwa, version 0.7.15-r1140 (http://bio-bwa.sourc eforge.net) (Li and Durbin, 2009),

Samtools, version 1.5 (http://samtools.sourceforge. net) (Li et al. 2009), 
Breakdancer, version v1.4.5 (https://github.com/ genome/breakdancer) (Chen et al. 2009), Perl, version v5.22.1. (https://www.perl.org/), FastQC, version fastqc_v0.11.5 (https://www.bioin formatics.babraham.ac.uk/projects/fastqc/), Hisat2, version 2.1.0 (https://daehwankimlab.github. io/hisat2/).(Kim et al. 2015),

HTSeq, version 0.11.1 (https://htseq.readthedocs.io/ en/release_0.11.1/count.html). (Anders et al. 2015), and DESeq2, (Love et al. 2014).

\section{Statistical analysis}

Statistical analyses were performed using Excel and R software (http://www.R-project.org/).

\section{Results}

QS-related phenotypes in P. aeruginosa laboratory strains. To investigate the QS characteristics in P. aeruginosa laboratory strains, we examined QS-related phenotypes of PAO1 strains commonly used in different labs. We tested three laboratory PAO1 strains, designated as PAO1-m, PAO1-u and PAO1-z. They were maintained in LB nutrition-rich broth, in which PAO1 generally is thought not to be subjected to selection pressures. The skim milk plate assay was used to assess their protease activity, which is mainly controlled by the Las QS system and partially by the Rhl QS system (Pearson et al.
1997). Although all of the strains showed protease-positive activity in the skim milk plate, PAO1-z displayed a smaller protease-catalyzed zoom than that in the PAO1$\mathrm{m}$ or the PAO1-u strain (Fig. 1A). This reduced proteolytic activity of PAO1-z implied that its QS activity was compromised to some degree, presumably resulting from genetic mutations associated with QS-related genes.

We therefore sequenced the component genes of the Las and Rhl QS systems in the PAO1-z strain, and found that the lasR gene has a 3-bp insertion in the ligand-binding domain (LBD) (herin referred to as LasR3 variant), resulting in a disrupted open reading frame (Fig. 1C). Furthermore, the Las QS reporter result revealed that the fluorescence level of PlasI-GFP in PAO1-z was as low as the PAO1-u-LasR mutant (Fig. 1B). Therefore, the LasR3 variant protein in PAO1-z was completely inactive. In addition, when the full-length of lasR 3 was deleted from PAO1-z, the resulting PAO1-z-LasR3 mutant still remained the similar proteolytic activity as the parent PAO1-z strain (Fig. 1D), suggesting LasR-3 variant in PAO1-z does not contribute to either the active proteolysis or the active QS system. Given that LasR is atop of the QS hierarchy (Lee and Zhang 2015), this finding raises the question how the production of QS-regulated protease was restored in the PAO1-z strain. Taken together, these findings show that different laboratory PAO1 strains have developed distinct QS phenotypes resulting from respective genome innovations. The Las QS activity in the PAO1-z strain was functionally disrupted due to a
A

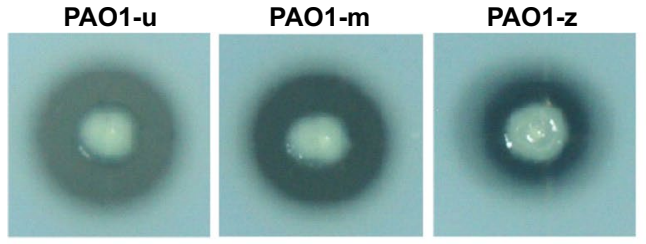

C

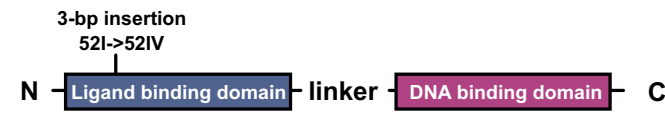

B

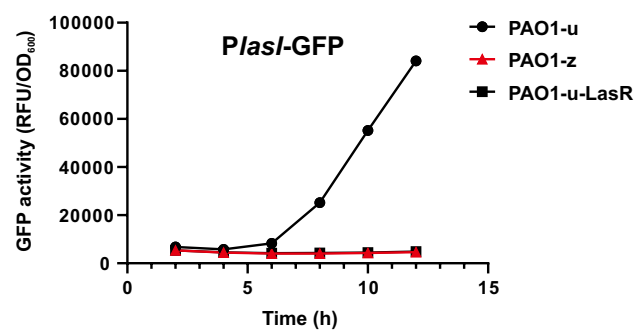

D

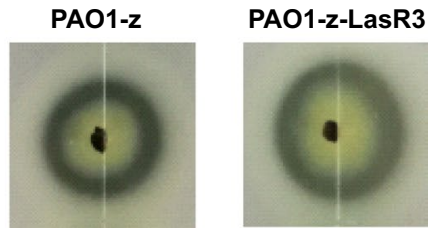

Fig. 1 QS activity profiles in P. aeruginosa laboratory strains. A Protease activity is shown in skim milk assay of three $P$. aeruginosa laboratory strains. An equal amount of bacteria were spotted on to the skim milk plate and photographed after $18 \mathrm{~h}$ of incubation. $\mathbf{B}$ The LasR-responsive activity in PAO1-u strain, PAO1-z strain and PAO1-u-LasR mutant. LasR-responsive activity was reflected by the fluorescence level of the Plas/-GFP reporter. The expression level of GFP in the tested strain was measured by the microplate reader and reported as relative fluorescence units (RFU). $\mathbf{C}$ IIlustration of 3-bp insertion in the ligand-binding domain of the lasR gene in the PAO1-z strain. D Protease activity of PAO1-z strain and PAO1-z-LasR3 mutant in skim milk plate. PAO1-z-LasR3 mutant, PAO1-z strain with deleting the lasR3 variant 
3-bp insertion occuring in the lasR gene, and the retained QS-controlled proteolysis implies that the QS hierarchy was re-adapted in the PAO1-z strain.

\section{Inactivating MexT is responsible for rewired QS phenotypes in PAO1-z}

To identify the genetic changes responsible for the altered QS phenotypes in PAO1-z, we performed the whole-genome short read re-sequencing (WGS) analysis. Short reads of PAO1-z were mapped to the reference PAO1 genome (NCBI accession number: NC_002516.2). Relative to the PAO1-u genome, PAO1-z has only a few genomic differences, including single-nucleotide substitutions, insertions and deletions. They include a 3-bp insertion in the lasR gene, an 18-bp deletion in the mexT gene, three single-nucleotide substitutions occurring in the tsap gene, gtrS gene and the noncoding region, respectively (Table 1 ). These DNA elements bearing mutations are thus the potential candidates responsible for the altered QS phenotypes in PAO1-z.
Because either overexpression or deletion of the mexT gene has been shown to substantially influence Rhl QS activity (Köhler et al. 2001; Tian et al. 2009b), we hypothesized that the 18-bp-deleted mexT gene in PAO1-z may encode a nonfunctional protein. In the skim milk plate assay, a functional mexT copy from PAO1-u was transferred into PAO1-z, resulting in a protease-deficient phenotype in the recombinant strain (Fig. 2A). This complementation result indicated the 18-bp-deleted mexT gene in PAO1-z was functionally inactive. Furthermore, this copy of mexT gene from PAO1-z was mobilized into PAO1-u-LasR-MexT double mutant but did not affect the protease activity in the resultant strain (Additional file 1: Figure S1). All these results indicated that the mexT gene in PAO1-z encodes a nonfunctional protein. Similar to the LasR-MexT mutant, PAO1-z also showed increased Rhl QS activity, as reflected by the PrhlA-GFP reporter (Fig. 2B) and elevated productions of pyocyanin pigment, cyanide and elastase when compared to the LasR mutant (Additional file 1: Figure S2). Shown by selected QS genes, the qRT-PCR analysis also confirmed

Table 1 Identification of genome mutations in the PAO1-z strain

\begin{tabular}{|c|c|c|c|c|c|c|}
\hline $\begin{array}{l}\text { Nucleotide } \\
\text { change }\end{array}$ & $\begin{array}{l}\text { Amino acid } \\
\text { change }\end{array}$ & Mutation type & Encoding & Targeted gene & Locus tag & Product \\
\hline A1558324 $\rightarrow$ ATCG & $521 \rightarrow I V$ & 3-bp insertion & CDS & lasR & PA1430 & $\begin{array}{l}\text { Transcriptional } \\
\text { regulator }\end{array}$ \\
\hline $\mathrm{C} 22278 \rightarrow \mathrm{T}$ & No change & SNP & CDS & tsaP & PA0020 & $\begin{array}{l}\text { T4P secretin- } \\
\text { associated } \\
\text { protein }\end{array}$ \\
\hline $\mathrm{C} 3582640 \rightarrow \mathrm{A}$ & $157 \mathrm{P} \rightarrow \mathrm{Q}$ & SNP & CDS & gtrs & PA3191 & $\begin{array}{l}\text { Glucose trans- } \\
\text { port sensor }\end{array}$ \\
\hline $\begin{array}{l}\text { GCGCTGTCG } \\
\text { CGCCTGCGC } \\
\text { A2807724 } \rightarrow \text { G }\end{array}$ & $\begin{array}{l}\text { Truncated } \\
\text { protein }\end{array}$ & 18-bp deletion & CDS & mext & PA2492 & $\begin{array}{l}\text { Transcriptional } \\
\text { regulator }\end{array}$ \\
\hline C5036907-> G & & SNP & Noncoding & & & \\
\hline
\end{tabular}

Whole-genome re-sequencing (WGS) was performed with the PAO1-z strain. Short reads were mapped to the reference PAO1 genome (NC_002516.2). Genome mutations were identified relative to the PAO1-u strain

A

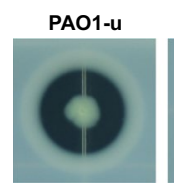

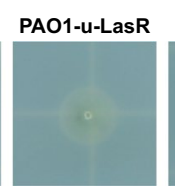
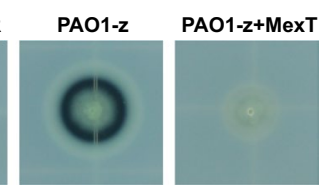

B

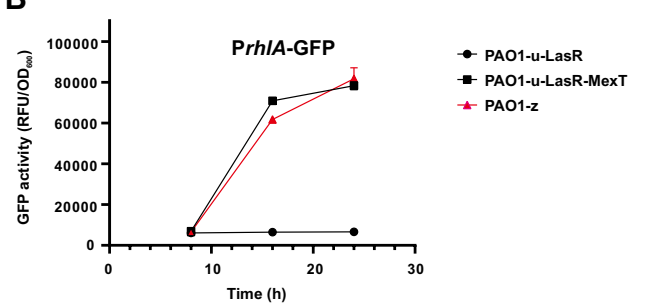

Fig. 2 Inactivating MexT responsible for the active Rhl QS phenotypes in PAO1-z. A Skim milk plate assay of indicated strains. The same amounts of bacteria were spotted onto the skim milk plate and photographed after $18 \mathrm{~h}$ incubation. B Detection of fluorescence of Prh/A-GFP reporter in indicated strains. The expression level of GFP in the tested strain was measured by the microplate reader and reported as relative fluorescence units (RFU). PAO1-u-LasR, PAO1-u strain with deleting the full-length of the lasR gene; PAO1-z+ MexT, PAO1-z strain was complemented with a construct expressing the mexT gene from the PAO1-u strain; PAO1-u-LasR-MexT, PAO1-u strain with deleting both of the lasR and mexT genes 
the elevated Rhl QS activity as well as the PQS QS activity in the PAO1-z strain (Additional file 1: Figure S3). Therefore, we concluded that PAO1-z has an inactive Las QS system, but the Rhl QS system is restored by the mexT mutation.

We did not further investigate the roles of the tsap gene and the gtrS gene for the QS adaptation in PAO1z. The single-nucleotide substitution in the tsap gene did not affect the coding amino acids (Table 1). Meanwhile, the gtrS genes encode a glucose transport sensor, which is less likely to be involved in the QS pathway regulation. We therefore reasoned that the lasR and mexT genes are most likely responsible for the evolution of QS adaptation in PAO1-z. Our study suggests that under certain laboratory conditions, the mutation that occurred in the lasR gene resulted in the inactivation of the LasR QS system, followed by the adaptive mutation in the mexT gene leading to the activation of the Rhl QS system.

\section{Discussion}

In our study, the adaptive mutations in the las $R$ and the mexT gene, which are responsible for the changes of the QS hierarchy, were identified in a commonly used laboratory $\mathrm{PAO} 1-\mathrm{z}$ strain cultured in nutrition-rich conditions. We assume that the lasR mutations inactivated QS first, followed by a secondary mexT mutation that reprogramed the QS hierarchy in the PAO1-z strain. It is very unlikely an opposite scenario of mutation occurred. $P$. aeruginosa has complex regulatory networks (Huang et al. 2019; Lee and Zhang 2015) that allow this bacterium to rearrange its QS hierarchy, and our findings demonstrate that $P$. aeruginosa could utilize QS to efficiently adapt to environments. QS homeostasis has previously been observed in LasR mutant cells during the stationary phase or under the starvation condition. In the late stationary phase, Rhl and PQS QS systems could achieve to be self-active in the LasR-null mutant (Dekimpe and Deziel 2009; Diggle et al. 2003), while LasR mutant cultured in protein-based broth could also emerge mexT mutations to activate the LasR-independent $\mathrm{Rhl}$ and PQS QS systems (Kostylev et al. 2019; Oshri et al. 2018). In comparison, our study found that the PAO1-z strain maintained in nutrient-rich broth also underwent genome variations that resulted in QS hierarchy autoregulation. Future research will be needed to fully elucidate the specific condition that could induce the QS autoregulation in the PAO1-z strain.

With adaption to the deteriorating environments in CF lungs, $P$. aeruginosa isolates in chronic infection appear evolutionary changes, resulting in a wide spectrum of colony variants that are hypermutable, nonmotile, nonflagellated, liposaccharide-deficient, resistant to antibiotics, auxotrophic (Oliver et al. 2000; Winstanley et al. 2016). Owing to high genetic and phenotypic diversities, genetic knowledge in clinical isolates is difficult to be translated into the understanding of the corresponding adapted phenotypes. In $P$. aeruginosa $\mathrm{CF}$ chronical infection isolates, mutations in the QS master regulator lasR gene were commonly identified (D'Argenio et al. 2007; Hoffman et al. 2009; Smith et al. 2006). Although the lasR mutation usually yields a nonfunctional protein causing the paralysis of the QS system (Feltner et al. 2016), LasR-null isolates were capable of engaging in QS activity with producing QS-associated factors and QS signal molecules (Bjarnsholt et al. 2010; Chen et al. 2019; Cruz et al. 2020; Feltner et al. 2016). Although the microevolution has been reported across laboratory PAO1 strains worldwide (Chandler et al. 2019; Klockgether et al. 2010), the frequency of mutation in laboratory PAO1 strains was rather lower than that in clinical isolates. This observation was supported in our WGS data with the identification of only a few adaptative mutations in the long-term laboratorymaintained PAO1-z strain (Table 1). Meanwhile, our findings also demonstrate that the laboratory-adapted strain experienced QS adaptation. Therefore, by examining the respective genotype-phenotype association, the laboratory PAO1 strain could serve as a useful platform for studying Pseudomonas QS adaptation by subjecting the bacterium to conditions similar to those found in clinical settings.

Our study found that the laboratory strain PAO1-z underwent genome innovations that led to the alteration of QS hierarchy. In such situation, the QS characteristics of a laboratory PAO1 strain may probably have been masked to some extent. This will impact on and mislead the following QS-related researches if using the QS-altered PAO1 strains. Our findings suggest that as a first step toward a proper illustration of QS adaptability in PAO1 strains, it is crucial to verify the potential effects derived from their genome variations.

\section{Supplementary Information}

The online version contains supplementary material available at https://doi. org/10.1186/s13568-022-01344-7.

Additional file 1. Supplementary information in this study.

\section{Acknowledgements}

We are grateful to E. Peter Greenberg (University of Washington, US) for giving worthful suggestions on this project. We also want to express our gratitude to lab members for their assistances and supports with respect to many aspects of this work. 


\section{Authors' contributions}

$X C, M L, Y L, H Q, L H$ and $W D$ designed experiments. XC, ML, YL, HQ conducted the experiments. WD performed bioinformatic analyses. WD wrote the manuscript. All authors read and approved the final manuscript.

\section{Funding}

This work was supported by the National Natural Science Foundation of China (31771341) and by Guangdong Province Science and Technology Innovation Strategy Special Fund (Grant No: 2018B020206001).

\section{Availability of data and materials}

The short-read sequencing data set have been deposited in the Sequence Read Archive (SRA) (www.ncbi.nlm.nih.gov/sra) under accession number PRJNA779485.

\section{Code availability}

Used software were listed in the section of Materials and methods.

\section{Declarations}

\section{Ethics approval and consent to participate}

This article does not contain any studies with human participants or animals performed by any of the authors.

\section{Consent for publication}

All authors have read and approved the final manuscript.

\section{Competing interests}

The authors declare that there are no conflicts of interest.

\section{Author details}

${ }^{1}$ Guangdong Laboratory for Lingnan Modern Agriculture, South China Agricultural University, Guangzhou 510642, China. ${ }^{2}$ Guangdong Province Key Laboratory of Microbial Signals and Disease Control, Integrative Microbiology Research Center, South China Agricultural University, Guangzhou 510642, China. ${ }^{3}$ Department of Biomedical Sciences, City University of Hong Kong, Kowloon, Hong Kong SAR, China. ${ }^{4}$ Division of Natural and Applied Sciences, Duke Kunshan University, Kunshan, Jiangsu, China.

Received: 22 December 2021 Accepted: 1 January 2022 Published online: 26 January 2022

\section{References}

Bjarnsholt T, Jensen $P \varnothing$, Jakobsen TH, Phipps R, Nielsen AK, Rybtke MT, TolkerNielsen T, Givskov M, Høiby N, Ciofu O (2010) Quorum sensing and virulence of Pseudomonas aeruginosa during lung infection of cystic fibrosis patients. PLoS ONE 5(4):e10115

Chandler CE, Horspool AM, Hill PJ, Wozniak DJ, Schertzer JW, Rasko DA, Ernst RK (2019) Genomic and phenotypic diversity among ten laboratory isolates of Pseudomonas aeruginosa PAO1. J Bacteriol 201(5):e00595-e618

Chen R, Déziel E, Groleau M-C, Schaefer AL, Greenberg EP (2019) Social cheating in a Pseudomonas aeruginosa quorum-sensing variant. PNAS 116(14):7021-7026

Choi K-H, Schweizer HP (2006a) mini-Tn7 insertion in bacteria with single attTn7 sites: example Pseudomonas aeruginosa. Nat Protoc 1(1):153

Choi K-H, Schweizer HP (2006b) mini-Tn 7 insertion in bacteria with single att Tn 7 sites: example Pseudomonas aeruginosa. Nat Protoc 1(1):153

Cruz RL, Asfahl KL, Van den Bossche S, Coenye T, Crabbé A, Dandekar AA (2020) RhIR-regulated acyl-homoserine lactone quorum sensing in a cystic fibrosis isolate of Pseudomonas aeruginosa. Mbio 11:2

D'Argenio DA, Wu M, Hoffman LR, Kulasekara HD, Déziel E, Smith EE, Nguyen H, Ernst RK, Larson Freeman TJ, Spencer DH (2007) Growth phenotypes of Pseudomonas aeruginosa lasR mutants adapted to the airways of cystic fibrosis patients. Mol Microbiol 64(2):512-533

Dekimpe V, Deziel E (2009) Revisiting the quorum-sensing hierarchy in Pseudomonas aeruginosa: the transcriptional regulator $\mathrm{RhIR}$ regulates LasR-specific factors. Microbiology 155(3):712-723
Diggle SP, Winzer K, Chhabra SR, Worrall KE, Cámara M, Williams P (2003) The Pseudomonas aeruginosa quinolone signal molecule overcomes the cell density-dependency of the quorum sensing hierarchy, regulates rhldependent genes at the onset of stationary phase and can be produced in the absence of LasR. Mol Microbiol 50(1):29-43

Feltner JB, Wolter DJ, Pope CE, Groleau M-C, Smalley NE, Greenberg EP, Mayer-Hamblett N, Burns J, Déziel E, Hoffman LR (2016) LasR variant cystic fibrosis isolates reveal an adaptable quorum-sensing hierarchy in Pseudomonas aeruginosa. Mbio 7(5):e01513-e1516

Gellatly SL, Hancock RE (2013) Pseudomonas aeruginosa: new insights into pathogenesis and host defenses. Pathog Dis 67(3):159-173

Gilbert J, Olivas A, Shogan Bd, Valuckaite V, Zaborin A, Belogortseva N, Musch M, Meyer F, WI T, An G (2012) Intestinal tissues induce an SNP mutation in pseudomonas aeruginosa that enhances its virulence: possible role in anastomotic leak. PLOS ONE 7:5

Hoffman LR, Kulasekara HD, Emerson J, Houston LS, Burns JL, Ramsey BW, Miller SI (2009) Pseudomonas aeruginosa lasR mutants are associated with cystic fibrosis lung disease progression. J Cyst Fibros 8(1):66-70

Holloway B (1955) Genetic recombination in Pseudomonas aeruginosa. Microbiology 13(3):572-581

Holloway AB, Morgan A, (1986) Genome organization in Pseudomonas. Ann Rev Microbiology 40(1):79-105

Huang H, Shao X, Xie Y, Wang T, Zhang Y, Wang X, Deng X (2019) An integrated genomic regulatory network of virulence-related transcriptional factors in Pseudomonas aeruginosa. Nat Commun 10(1):1-13

Klockgether J, Tümmler B (2017) Recent advances in understanding Pseudomonas aeruginosa as a pathogen. F1000Research 6:1000

Klockgether J, Munder A, Neugebauer J, Davenport CF, Stanke F, Larbig KD, Heeb S, SchöckPohIWiehlmann UTML (2010) Genome diversity of Pseudomonas aeruginosa PAO1 laboratory strains. J Bacteriol 192(4):1113-1121

Ko" hler T, Michéa-Hamzehpour M, Henze U, Gotoh N, Kocjancic Curty L, Pechère JC, (1997) Characterization of MexE-MexF-OprN, a positively regulated multidrug efflux system of Pseudomonas aeruginosa. Mol Microbiol 23(2):345-354

Köhler T, Michea-Hamzehpour M, Plesiat P, Kahr A-L, Pechere J-C (1997) Differential selection of multidrug efflux systems by quinolones in Pseudomonas aeruginosa. Antimicrob Agents Chemother 41(11):2540-2543

Köhler T, Epp SF, Curty LK, Pechère J-C, (1999) Characterization of MexT, the regulator of the MexE-MexF-OprN multidrug efflux system of Pseudomonas aeruginosa. J Bacteriol 181(20):6300-6305

Köhler T, Van Delden C, Curty LK, Hamzehpour MM, Pechere J-C, (2001) Overexpression of the MexEF-OprN multidrug efflux system affects cell-to-cell signaling in Pseudomonas aeruginosa. J Bacteriol 183(18):5213-5222

Kostylev M, Kim DY, Smalley NE, Salukhe I, Greenberg EP, Dandekar AA (2019) Evolution of the Pseudomonas aeruginosa quorum-sensing hierarchy. PNAS 116(14):7027-7032

Lamarche MG, Déziel E (2011e) MexEF-OprN efflux pump exports the Pseudomonas quinolone signal $(\mathrm{PQS})$ precursor HHQ (4-hydroxy-2-heptylquinoline). PLOS ONE 6(9):e24310

Lee J, Zhang L (2015) The hierarchy quorum sensing network in Pseudomonas aeruginosa. Protein Cell 6(1):26-41

Luong PM, Shogan BD, Zaborin A, Belogortseva N, Shrout JD, Zaborina O, Alverdy JC (2014) Emergence of the P2 phenotype in Pseudomonas aeruginosa PAO1 strains involves various mutations in mexT or mexF. J Bacteriol 196(2):504-513

Maddocks SE, Oyston PC (2008) Structure and function of the LysRtype transcriptional regulator (LTTR) family proteins. Microbiology 154(12):3609-3623

Maseda H, Saito K, Nakajima A, Nakae T (2000) Variation of the mexT gene, a regulator of the MexEF-oprN efflux pump expression in wild-type strains of Pseudomonas aeruginosa. FEMS Microbiol Lett 192(1):107-112

Oliver A, Cantón R, Campo P, Baquero F, Blázquez J (2000) High frequency of hypermutable Pseudomonas aeruginosa in cystic fibrosis lung infection. Science 288(5469):1251-1253

Oshri RD, Zrihen KS, Shner I, Bendori SO, Eldar A (2018) Selection for increased quorum-sensing cooperation in Pseudomonas aeruginosa through the shut-down of a drug resistance pump. ISME J 12(10):2458-2469

Papenfort K, Bassler BL (2016) Quorum sensing signal-response systems in Gram-negative bacteria. Nat Rev Microbiol 14(9):576 
Pearson JP, Pesci EC, Iglewski BH (1997) Roles of Pseudomonas aeruginosa las and rhl quorum-sensing systems in control of elastase and rhamnolipid biosynthesis genes. J Bacteriol 179(18):5756-5767

Rietsch A, Vallet-Gely I, Dove SL, Mekalanos JJ (2005) ExsE, a secreted regulator of type III secretion genes in Pseudomonas aeruginosa. PNAS 102(22):8006-8011

Smith EE, Buckley DG, Wu Z, Saenphimmachak C, Hoffman LR, D'Argenio DA, Miller SI, Ramsey BW, Speert DP, Moskowitz SM (2006) Genetic adaptation by Pseudomonas aeruginosa to the airways of cystic fibrosis patients. PNAS 103(22):8487-8492

Stover CK, Pham XQ, Erwin A, Mizoguchi S, Warrener P, Hickey M, Brinkman F, Hufnagle W, Kowalik D, Lagrou M (2000) Complete genome sequence of Pseudomonas aeruginosa PAO1, an opportunistic pathogen. Nature 406(6799):959-964

Tian Z-X, Fargier E, Mac Aogain M, Adams C, Wang Y-P, O'Gara F (2009a) Transcriptome profiling defines a novel regulon modulated by the LysR-type transcriptional regulator MexT in Pseudomonas aeruginosa. Nucleic Acids Res 37(22):7546-7559

Tian Z-X, Mac Aogain M, O'Connor HF, Fargier E, Mooij MJ, Adams C, Wang Y-P, O'Gara F (2009b) MexT modulates virulence determinants in Pseudomonas aeruginosa independent of the MexEF-OprN efflux pump. Microb Pathog 47(4):237-241

Winstanley C, O'Brien S, Brockhurst MA (2016) Pseudomonas aeruginosa evolutionary adaptation and diversification in cystic fibrosis chronic lung infections. Trends Microbiol 24(5):327-337

\section{Publisher's Note}

Springer Nature remains neutral with regard to jurisdictional claims in published maps and institutional affiliations.

\section{Submit your manuscript to a SpringerOpen ${ }^{\odot}$ journal and benefit from:}

- Convenient online submission

- Rigorous peer review

- Open access: articles freely available online

- High visibility within the field

- Retaining the copyright to your article 